\title{
Front Matter: Volume 7587
}

, "Front Matter: Volume 7587," Proc. SPIE 7587, Free-Space Laser Communication Technologies XXII, 758701 (12 March 2010); doi: 10.1117/12.862344

SPIE. Event: SPIE LASE, 2010, San Francisco, California, United States 


\section{PROCEEDINGS OF SPIE}

\section{Free-Space Laser Communication Technologies XXII}

Hamid Hemmati

Editor

26-28 January 2010

San Francisco, California, United States

Sponsored and Published by

SPIE 
The papers included in this volume were part of the technical conference cited on the cover and title page. Papers were selected and subject to review by the editors and conference program committee. Some conference presentations may not be available for publication. The papers published in these proceedings reflect the work and thoughts of the authors and are published herein as submitted. The publisher is not responsible for the validity of the information or for any outcomes resulting from reliance thereon.

Please use the following format to cite material from this book:

Author(s), "Title of Paper," in Free-Space Laser Communication Technologies XXII, edited by Hamid Hemmati, Proceedings of SPIE Vol. 7587 (SPIE, Bellingham, WA, 2010) Article CID Number.

ISSN 0277-786X

ISBN 9780819479839

Published by

SPIE

P.O. Box 10, Bellingham, Washington 98227-0010 USA

Telephone +1 3606763290 (Pacific Time) · Fax +1 3606471445

SPIE.org

Copyright (c) 2010, Society of Photo-Optical Instrumentation Engineers

Copying of material in this book for internal or personal use, or for the internal or personal use of specific clients, beyond the fair use provisions granted by the U.S. Copyright Law is authorized by SPIE subject to payment of copying fees. The Transactional Reporting Service base fee for this volume is $\$ 18.00$ per article (or portion thereof), which should be paid directly to the Copyright Clearance Center (CCC), 222 Rosewood Drive, Danvers, MA 01923. Payment may also be made electronically through CCC Online at copyright.com. Other copying for republication, resale, advertising or promotion, or any form of systematic or multiple reproduction of any material in this book is prohibited except with permission in writing from the publisher. The CCC fee code is 0277-786X/10/\$18.00.

Printed in the United States of America.

Publication of record for individual papers is online in the SPIE Digital Library.

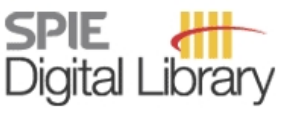

SPIEDigitalLibrary.org

Paper Numbering: Proceedings of SPIE follow an e-First publication model, with papers published first online and then in print and on CD-ROM. Papers are published as they are submitted and meet publication criteria. A unique, consistent, permanent citation identifier (CID) number is assigned to each article at the time of the first publication. Utilization of CIDs allows articles to be fully citable as soon they are published online, and connects the same identifier to all online, print, and electronic versions of the publication. SPIE uses a six-digit CID article numbering system in which:

- The first four digits correspond to the SPIE volume number.

- The last two digits indicate publication order within the volume using a Base 36 numbering system employing both numerals and letters. These two-number sets start with 00, 01, 02, 03, 04 , $05,06,07,08,09,0 A, 0 B \ldots$. OZ, followed by 10-1Z, 20-2Z, etc.

The CID number appears on each page of the manuscript. The complete citation is used on the first page, and an abbreviated version on subsequent pages. Numbers in the index correspond to the last two digits of the six-digit CID number. 


\section{Contents}

vii Conference Committee

ix Ultrafast fiber laser technology: status and prospects (Plenary Paper) [7579-102]

A. Tünnermann, J. Limpert, Friedrich-Schiller-Univ. Jena (Germany) and Fraunhofer-Institute for Applied Optics and Precision Engineering (Germany)

\section{SESSION 1 INVITED SESSION}

758702 Free space optical communications research at the U.S. Naval Research Laboratory (Invited Paper) [7587-01]

W. S. Rabinovich, C. I. Moore, H. R. Burris, J. L. Murphy, M. R. Suite, R. Mahon, M. S. Ferraro, P. G. Goetz, L. M. Thomas, C. Font, G. C. Gilbreath, B. XU, S. Binari, U.S. Naval Research Lab. (United States); K. Hacker, S. Reese, Naval Explosive Ordnance Disposal Technology Div. (United States); W. T. Freeman, S. Frawley, Smart Logic, Inc. (United States); E. Saint-Georges, S. Uecke, J. Sender, NOVASOL (United States)

758703 Preliminary results of the OCTL to OICETS optical link experiment (OTOOLE) (Invited Paper) [7587-02]

K. E. Wilson, J. Kovalik, A. Biswas, M. Wright, W. T. Roberts, Jet Propulsion Lab. (United States);

Y. Takayama, National Institute of Information and Communications Technology (Japan);

S. Yamakawa, Japan Aerospace Exploration Agency (Japan)

758704 Lasercom for interplanetary missions: recent European activities, future possibilities, and synergy aspects (Invited Paper) [7587-03]

T. Dreischer, F. Arnold, K. Kudielka, Y. Tissot, T. Weigel, RUAG Space AG (Switzerland)

758705 Optical satellite communications in Europe (Invited Paper) [7587-04]

Z. Sodnik, H. Lutz, B. Furch, European Space Agency (Netherlands); R. Meyer, German Aerospace Ctr. (Germany)

\section{SESSION 2 DEMONSTRATIONS}

758707 Free-space gigabit laser link experiment incorporating Japanese and Canadian technology development [7587-06]

A. Koujelev, D. Gratton, L. Hotte, Canadian Space Agency (Canada); Y. Arimoto, National Institute of Information and Communications Technology (Japan)

758708 Near field laser transmission with bidirectional beacon tracking for Tbps class wireless communications [7587-07]

Y. Arimoto, National Institute of Information and Communications Technology (Japan) 
758709 Laser communications for unmanned aircraft systems using differential GPS and IMU data [7587-08]

M. Czarnomski, J. Blakely, Z. Wang, G. Williamson, R. R. Schultz, W. H. Semke, Univ. of North Dakota (United States)

7587 OA Precision optical ranging by paired one-way time of flight [7587-09]

K. M. Birnbaum, Y. Chen, H. Hemmati, Jet Propulsion Lab. (United States)

7587 OB Emulating an optical planetary access link with an aircraft [7587-10]

A. Biswas, J. Kovalik, M. W. Regehr, M. Wright, Jet Propulsion Lab. (United States)

7587 OC Data products for the OCTL to OICETS optical link experiment [7587-11]

J. Kovalik, A. Biswas, K. Wilson, M. Wright, W. T. Roberts, Jet Propulsion Lab. (United States)

7587 OD Expanded laser communications demonstrations with OICETS and ground stations [7587-12] Y. Takayama, M. Toyoshima, Y. Shoji, Y. Koyama, H. Kunimori, National Institute of Information and Communications Technology (Japan); M. Sakave, S. Yamakawa, Japan Aerospace Exploration Agency (Japan); Y. Tashima, N. Kura, Space Engineering Development (Japan)

7587 OE Inter-satellite and satellite-ground laser communication links based on homodyne BPSK [7587-13]

M. Gregory, F. Heine, H. Kämpfner, R. Lange, K. Saucke, U. Sterr, Tesat-Spacecom

(Germany); R. Meyer, German Aerospace Ctr. (Germany)

\section{SESSION 3 TECHNOLOGIES: FLIGHT AND GROUND I}

7587 OF Experimental demonstration of a retro-reflective laser communication link on a mobile platform [7587-14]

V. V. Nikulin, Binghamton Univ. (United States); J. E. Malowicki, Air Force Research Lab. (United States); R. M. Khandekar, V. A. Skormin, Binghamton Univ. (United States);

D. J. Legare, Air Force Research Lab. (United States)

7587 OG High energy laser testbed for accurate beam pointing control [7587-15]

D. Kim, Agency for Defense Development (Korea, Republic of); J. J. Kim, D. Frist, M. Nagashima, B. Agrawal, Naval Postgraduate School (United States)

$7587 \mathrm{OH}$ Low complexity transceivers and autonomous concept of operations for optical planetary access links [7587-16]

A. Biswas, J. Kovalik, M. Wright, M. Regehr, Jet Propulsion Lab. (United States)

$7587 \mathrm{Ol}$ Modeling and measurement of effects of atmospheric turbulence and plafform jitter on free-space laser communication [7587-17]

Z. Liu, Binghamton Univ. (United States) and Case Western Reserve Univ. (United States);

V. V. Nikulin, R. Khandekar, Binghamton Univ. (United States)

7587 OK Low voltage actuator using carbon nanotube to tilt mirror angle [7587-19]

Y. Takayama, M. Toyoshima, National Institute of Information and Communications

Technology (Japan); T. Abe, Space Link Co. Ltd. (Japan) 
7587 OM Transmitter and translating receiver design for 64-ary pulse position modulation (PPM) [7587-21]

A. J. Mendez, Mendez R\&D Associates (United States); V. J. Hernandez, Lawrence Livermore National Lab. (United States); R. M. Gagliardi, The Univ. of Southern California (United States); C. V. Bennett, Lawrence Livermore National Lab. (United States)

7587 ON A review of the information capacity of single-mode free-space optical communication [7587-22]

B. I. Erkmen, B. E. Moision, K. M. Birnbaum, Jet Propulsion Lab. (United States)

\section{SESSION 4 TECHNOLOGIES: FLIGHT AND GROUND II}

758700 A PSAM-based estimator of noise and fading statistics for optimum receivers of free space optics signals [7587-23]

H. Moradi, H. H. Refai, Univ. of Oklahoma (United States); P. G. LoPresti, The Univ. of Tulsa (United States); M. Atiquzzaman, Univ. of Oklahoma (United States)

7587 OP JAXA's efforts toward next generation space data-relay satellite using optical inter-orbit communication technology [7587-24]

S. Yamakawa, T. Hanada, H. Kohata, Y. Fujiwara, Japan Aerospace Exploration Agency (Japan)

$75870 Q$ Separating and tracking multiple beacon sources for deep space optical communications [7587-25]

K. M. Birnbaum, A. Sahasrabudhe, W. H. Farr, Jet Propulsion Lab. (United States)

7587 OR High-efficiency high-power fiber master oscillator power amplifier for deep-space communication operating at $1532 \mathrm{~nm}$ [7587-26]

D. Sipes, Optical Engines, Inc. (United States); A. L. Moretti, X. Luo, A. R. Sugg, Vega Wave Systems, Inc. (United States)

7587 OS Free space optical communication utilizing mid-infrared interband cascade laser [7587-27] A. Soibel, M. Wright, W. Farr, S. Keo, C. Hill, Jet Propulsion Lab. (United States); R. Q. Yang, Univ. of Oklahoma (United States); H. C. Liu, National Research Council Canada (Canada)

7587 OT Challenges of developing resonant cavity photon-counting detectors at $1064 \mathbf{n m}$ [7587-28] S. Vasile, aPeak, Inc. (United States); M. S. Ünlü, Boston Univ. (United States); J. Lipson, aPeak, Inc. (United States)

7587 OU Study on the fiber coupling efficiency for ground-to-satellite laser communication links [7587-29]

H. Takenaka, National Institute of Information and Communications Technology (Japan) and Univ. of Electro-Communications (Japan); M. Toyoshima, National Institute of Information and Communications Technology (Japan)

\section{SESSION 5 CONCEPTS AND ANALYSIS}

$7587 \mathrm{OV}$ Stray light modeling and performance of the $15 \mathrm{~cm}$ deep space optical communications transceiver (DSOCT) [7587-30]

W. T. Roberts, Jet Propulsion Lab. (United States) 
7587 OW Intensity distribution based space and time division multiple access technique for hybrid-LOS indoor optical wireless communication [7587-31]

S. Miyamoto, K. Kawamoto, S. Sampei, Osaka Univ. (Japan)

7587 OX Toward an optimal combined FSO/RF system via an adaptive bit rate control [7587-32]

H. Moradi, M. Falahpour, H. H. Refai, Univ. of Oklahoma (United States); P. G. LoPresti, The Univ. of Tulsa (United States); M. Atiquzzaman, Univ. of Oklahoma (United States)

7587 OY OCTL to OICETS optical link experiment (OTOOLE) electrooptical systems [7587-34]

W. T. Roberts, M. W. Wright, J. Kovalik, V. Garkanian, K. E. Wilson, Jet Propulsion Lab. (United States)

Author Index 


\title{
Conference Committee
}

\author{
Symposium Chairs \\ Donald J. Harter, IMRA America, Inc. (United States) \\ Peter R. Herman, University of Toronto (Canada) \\ Symposium Cochairs
}

Alberto Piqué, Naval Research Laboratory (United States)

Friedhelm Dorsch, TRUMPF Photonics (United States)

Conference Chair

Hamid Hemmati, Jet Propulsion Laboratory (United States)

Program Committee

Don M. Boroson, MIT Lincoln Laboratory (United States)

Ronald W. Burch, The Boeing Company (United States)

Vincent W. Chan, Massachusetts Institute of Technology (United States)

Wayne R. Fenner, The Aerospace Corporation (United States)

G. Charmaine Gilbreath, Naval Research Laboratory (United States)

Michael A. Krainak, NASA Goddard Space Flight Center (United States)

Robert Lange, Tesat-Spacecom GmbH \& Company KG (Germany)

Zoran Sodnik, European Space Research and Technology Center (Netherlands)

Morio Toyoshima, National Institute of Information and

Communications Technology (Japan)

Alan E. Willner, University of Southern California (United States)

Shiro Yamakawa, Japan Aerospace Exploration Agency (Japan)

Session Chairs

1 Invited Session

Hamid Hemmati, Jet Propulsion Laboratory (United States)

2 Demonstrations

Abhijit Biswas, Jet Propulsion Laboratory (United States)

3 Technologies: Flight and Ground I

Joseph M. Kovalik, Jet Propulsion Laboratory (United States) 
4 Technologies: Flight and Ground II

Keith E. Wilson, Jet Propulsion Laboratory (United States)

5 Concepts and Analysis

Robert Lange, Tesat-Spacecom GmbH \& Company KG (Germany) 